\title{
Positional information in short-term memory: Relative or absolute?
}

\author{
RICHARD N. A. HENSON \\ University College London, London, England
}

\begin{abstract}
Evidence suggests that short-term memory for serial order includes information about the positions of items in a sequence. This information is necessary to explain why substitution errors between sequences tend to maintain their position within a sequence. Previous demonstrations of such errors, however, have always used sequences of equal length. With sequences of different length, both transpositions between groups (Experiment 1) and intrusions between trials (Experiment 2) are shown to respect position relative to the end as well as to the start of a sequence. These results support models in which position is coded by start and end markers, but not models in which position is coded in temporal or absolute terms. Possible interpretations of an end marker are discussed.
\end{abstract}

There is much recent interest in computational models of short-term memory (e.g., Anderson \& Matessa, 1997; Brown, Preece, \& Hulme, in press; Burgess \& Hitch, 1992; Henson, 1998b; Lee \& Estes, 1981; Lewandowsky \& Murdock, 1989; Page \& Norris, 1998; Shiffrin \& Cook, 1978). A key issue addressed by these models is the problem of serial order: that is, how people store and retrieve a novel sequence of items in the correct order. One solution to this problem is to assume that each item is coded for its position in the sequence (see, e.g., Anderson \& Matessa, 1997; Brown et al., in press; Burgess \& Hitch, 1992; Henson, 1998b; Lee \& Estes, 1981). The purpose of the present article is to further elucidate the nature of this positional information.

The presence of positional information in short-term memory is indicated by the occurrence of positional errors in serial recall. One type of positional error arises when stimuli are grouped. For example, a list of nine items might be grouped as three groups of three by the insertion of a pause after every third item (see, e.g., Ryan, 1969). Grouping improves memory for the order of items, foremost by reducing the number of transposition errors between groups. However, one type of transposition is increased by grouping: those transpositions between groups that maintain their position within groups (Wickelgren, 1967). With groups of three for example,

This paper is based on part of the author's doctoral dissertation at the University of Cambridge, England, which was supported by a Research Studentship from the Medical Research Council. The author thanks Alan Baddeley, Gordon Brown, Graham Hitch, Dennis Norris, and Mike Page for reading preliminary drafts, Ian Nimmo-Smith for statistical advice, and Robert Greene, Bennett Murdock, John Wixted, and two anonymous reviewers for helpful comments during review. The research was conducted at the MRC Applied Psychology Unit, Cambridge. The author has since moved to University College London. Correspondence concerning this article should be addressed to R. N. A. Henson, Wellcome Department of Cognitive Neurology, 12 Queen Square, London WC1N 3BG, England (e-mail: thenson@fil.ion.ucl.ac.uk). these errors are indicated by an increase in the number of three-apart transpositions. Henson (1996) called such errors interpositions and showed that they are not a result of whole groups swapping (see, also, Lee \& Estes, 1981; Nairne, 1991). Interpositions imply that items are coded for their position within a group independently of surrounding items.

A second type of positional error arises between trials. For example, Conrad (1960) showed that erroneous items were more likely to occur at the same position in the previous trial than would be predicted by chance (see, also, Estes, 1991). Henson (1996) called such errors protrusions and showed that they are more often from the previous recall episode than from the previous presentation episode (suggesting that recall is itself a recoding process). Protrusions imply that items are coded for their position within a list independently of surrounding items.

Interpositions and protrusions are examples of a general tendency for substitutions between sequences to maintain their position within a sequence. Models that simulate such errors assume that position is coded at multiple levels - such as position-in-group, position-inlist, and so forth-with positional errors arising between items that share the same code at one or more levels (see, e.g., Lee \& Estes, 1981). However, the nature of these positional codes remains unclear. Some theories assume that items are coded directly by their time of occurrence (e.g., Yntema \& Trask, 1963). Position can then be defined relative to the time of occurrence of the first item, as in models in which items are associated with the states of temporal oscillators (e.g., Brown et al., in press; Brown \& Vousden, 1998; Burgess \& Hitch, 1996). Alternatively, items can be associated with some representation of their ordinal position (i.e., first, second, third, etc.), regardless of their exact timing (see, e.g., Anderson \& Matessa, 1997; Burgess \& Hitch, 1992). All of these theories code position in absolute terms, in the sense that position is defined independently of the total number of items in a 
sequence: Assuming that the presentation rate is constant, the code for the third position in a sequence of three items is identical to the code for the third position in a sequence of four items.

Other models, however, code position relative to both the start and the end of a sequence (Henson, 1998b; Houghton, 1990). The model of Henson (1996, 1998b), for example, presumes a start marker and an end marker, the relative strengths of which provide an approximate code for each position within a sequence. This coding of relative position is distinguished from one of absolute position by being sensitive to sequence length: The code for the third position in a sequence of three items differs from that for the third position in a sequence of four items. ${ }^{1}$

Previous demonstrations of positional errors do not distinguish between absolute and relative position, because they use sequences of equal length, for which the two definitions of position are confounded. The solution is to examine the positional errors between sequences of different lengths. For example, consider transpositions between a group of three items followed by a group of four (upper illustration in Figure 1): Does the third and final item of the first group tend to swap with the third item of the second group or with the fourth and final item of the second group? The absolute interpositions in the former case are transpositions that respect absolute position within a group (i.e., third to third); the relative interpositions in the latter case are transpositions that respect relative position within a group (i.e., end to end). Or consider recall of a list of seven items on one trial, followed by recall of a list of five items on the next (lower illustration in Figure 1): Are most intrusions on the fifth

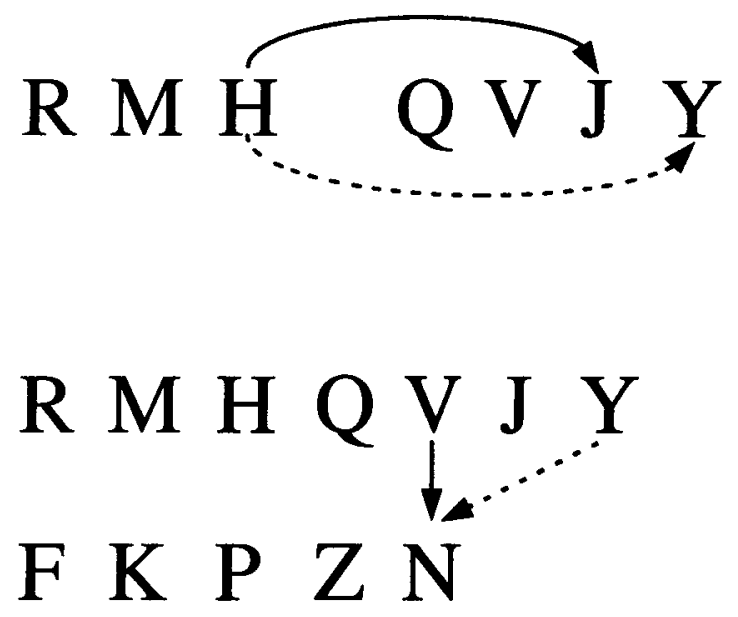

$\longrightarrow$ Absolute

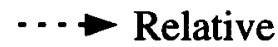

Figure 1. Absolute and relative positional errors. and final position of the report of five items from the fifth position of the previous report or from the seventh and final position of the previous report? In other words, which are more common: absolute protrusions or relative protrusions, respectively? These questions are addressed in the two experiments below.

\section{EXPERIMENT 1}

The first experiment tested whether transpositions between groups of different size maintain absolute or relative position within groups. In the grouped 3-4 condition, lists of seven items were split into a group of three followed by a group of four. The critical positions were Positions 3 and 6 (third positions within the groups) and Positions 3 and 7 (final positions within the groups). Absolute interpositions between the third position of groups were errors when Item 3 was recalled in Position 6 or Item 6 was recalled in Position 3. Relative interpositions between the ends of groups were errors when Item 7 was recalled in Position 3 or Item 3 was recalled in Position 7. In the grouped 4-3 condition, the lists were split into a group of four followed by a group of three. In this case, the critical positions were Positions 3 and 7 and Positions 4 and 7 . Absolute interpositions were errors when Item 3 was recalled in Position 7 or Item 7 was recalled in Position 3 , and relative interpositions were errors when Item 7 was recalled in Position 4 or Item 4 was recalled in Position 7. Finally, an ungrouped condition was included, to check that differences in relative and absolute interpositions were not simply an artifact of different baseline probabilities.

One additional manipulation in the present experiment was to ask the participants to indicate the confidence of each response. It is possible that positional errors might simply be the result of guessing strategies when recall fails. If so, positional errors should disappear once guesses are removed from the analysis. If positional errors remain, however, further support will be obtained for the integral role of positional information in shortterm memory.

\section{Method}

Participants. Twenty-four students from Cambridge University were tested, 12 male and 12 female, with a mean age of 20 years.

Materials. Three blocks of 30 lists were constructed. The lists were permutations of seven single-syllable words, drawn from the set goose, verve, latch, bathe, flown, clump, and trout. Such a small experimental vocabulary was chosen in order to minimize item errors in favor of order items (i.e., transpositions) and, hence, to maximize the incidence of interpositions. Each word had five letters, a Kučera-Francis (1967) frequency of between four and five, and both a unique vowel sound and a unique first letter. The order of words within lists was randomized, except for the constraint that, over a block of trials, each word appeared approximately equally often at each position.

Procedure. The blocks were assigned to three conditions in a counterbalanced fashion. In the ungrouped condition, the seven words were presented in the center of a VDU at a rate of just over one a second ( $600 \mathrm{msec}$ on, $200 \mathrm{msec}$ off), each word replacing its 
predecessor. In the grouped 3-4 condition, there was an additional $800-\mathrm{msec}$ pause between the third and the fourth words; in the grouped 4-3 condition, there was an additional $800-\mathrm{msec}$ pause between the fourth and the fifth words. The participants read the words in silence and were told to use the pause to group the lists appropriately. As soon as the last item had disappeared, a visual cue followed for immediate, serial recall.

The participants recalled the list by writing the first letter of each word in two rows of seven boxes provided on a response sheet, which were grouped appropriately for each condition. The requirement to write only the first letter of each word was chosen in order to minimize the output delay and, hence, ensure reasonable performance (see Baddeley, Thomson, \& Buchanan, 1975). The participants were told to write responses they were sure about in the top row and responses they were not sure about, or which were guesses, in the bottom row (cf. Henson, 1998a). They could move up and down the rows as much as they liked, as long as they gave one and only one response in each column (i.e., gave exactly seven responses in total). All seven words were permanently on display, from which the participants could guess, if necessary. The participants were asked to write from left to right on the response sheet, recalling the lists in a forward order. Although they only had to write the first letter of each word, the participants were told to remember the lists as lists of whole words (and all reported obeying this instruction).

The participants received six ungrouped practice trials, followed by the three blocks of lists. The order of blocks was counterbalanced across participants. The order of conditions was constrained by the fact that the ungrouped condition was always first, followed by the two grouped conditions, which alternated across participants. This was done to reduce the tendency for participants to group the ungrouped lists subjectively, as would be likely if a grouped condition preceded the ungrouped one (Henson, 1996). The experiment lasted $40 \mathrm{~min}$.

Hypothesis testing. Statistical tests in the present study were made on empirical log-odds, which were weighted for the purposes of pairwise comparisons (Cox \& Snell, 1989). It has been argued (Allerup \& Elbro, 1998) that the log-odds transform is the most appropriate transform for comparing proportional scores. In particular, the empiricalized log-odds transform makes some allowance for floor effects (see the Appendix), which is important for the present experiments where the proportions are small.

\section{Results}

In brief, relative interpositions between the ends of groups were more common than absolute interpositions between the third position of groups, regardless of whether guesses were included or excluded, although the difference was significant only in the grouped 3-4 condition. However, many relative interpositions were repetitions of an item at the end of both groups, and when analysis was confined to the second group, grouping had a clear effect of increasing relative interpositions over absolute interpositions.

Overall performance. The proportion of lists correct was greater in the grouped 3-4 $(M=.39, S D=.29)$ and grouped 4-3 $(M=.39, S D=.27)$ conditions than in the ungrouped condition $(M=.22, S D=.22)$. Tests of weighted log-odds showed that the difference was significant in both cases $[Z(24)>7.25, p<.0001]$, but there was no significant difference between the two styles of grouping $[Z(24)=0.09, p=.93]$. The serial position curves in Figure 2 suggest some spontaneous 4-3 grouping in the ungrouped condition, although several grouping strategies were reported (e.g., 3-4, 2-2-3, and 3-2-2). Guesses made up approximately $25 \%$ of the responses ( $50 \%$ of errors), on average, and removing guesses reduced the number of correct responses on all the positions (lower panel of Figure 2).

Errors on critical positions. The proportions of errors on a critical position that were interpositions from the other critical position are shown in Table 1 (with the same seven items every trial, the only errors made were transpositions). With guesses removed, the proportions were calculated from the number of transpositions remaining (leading to the exclusion of 2 participants who made no errors on critical positions, once guesses were removed). The proportions were small because the majority of transpositions on critical positions were from adjacent within-group positions (Henson, 1996). In order to compare these proportions with baseline measures in ungrouped lists, the ungrouped lists were scored as if they were grouped 3-4 or 4-3.

For 3-4 grouping, a three-way repeated measures analysis of variance (ANOVA) on empirical log-odds showed a significant interaction between position in group (third vs. final) and condition (grouped vs. ungrouped) $[F(1,21)=8.89, p<.01]$. The effect of position approached significance $[F(1,21)=4.34, p=.05]$, but there was no significant effect of condition $[F(1,21)<1]$ or guessing (including vs. excluding guesses) $[F(1,21)=$ $2.17, p=.16]$, or any other significant interactions $[F(1,21)<1.06, p>.31]$. The effect of position reflected a greater incidence of interpositions between final positions than between third positions, and the interaction with condition showed that this difference was greater in the grouped than in the ungrouped condition. In other words, interpositions respected relative rather than absolute position within groups, and this difference did not appear to be an artifact of different baseline probabilities in ungrouped lists.

For 4-3 grouping, the corresponding ANOVA did not show a significant interaction between position and condition $[F(1,21)=2.02, p=.17]$, or any other significant effects or interactions $[F(1,21)<1.75, p>.20]$. Despite the lack of statistical significance, however, the pattern of results was very similar to that for 3-4 grouping. A reason for the difference in the size of effects in the grouped 3-4 and grouped 4-3 conditions is given in the Discussion section.

One possible confound of the above analyses concerns items transposed from the group of four to the group of three. ${ }^{2}$ In these cases, one might argue that the analyses are biased against absolute interpositions, because there is no fourth position in the group of three that corresponds to the fourth position in the group of four. In other words, although an error on the third position in the group of three that came from the fourth position in the group of four would be scored as a relative interposition, it could also reflect the fact that the item in the fourth position of the group of four has no corresponding absolute position in which to be recalled. To counter this pos- 

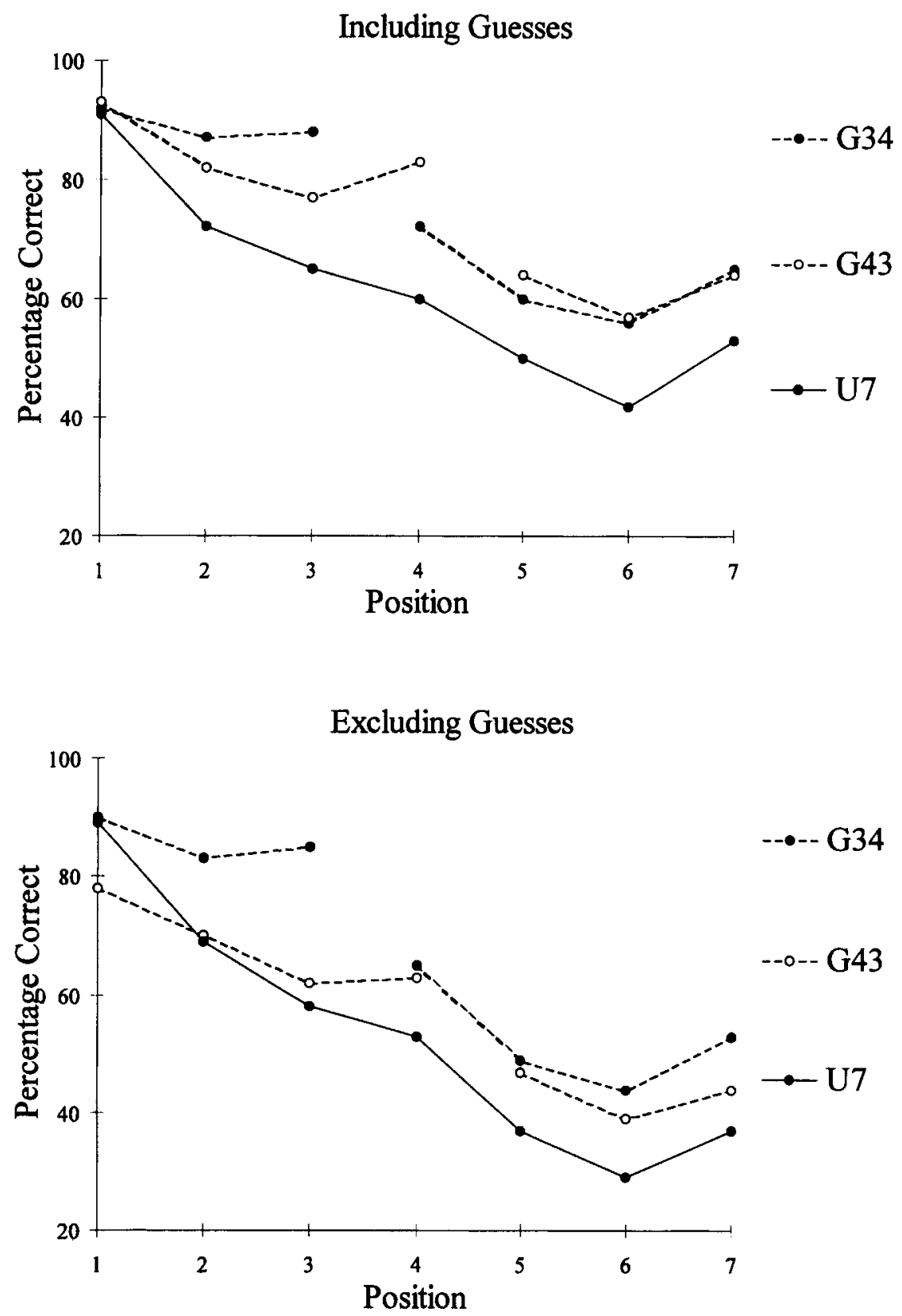

Figure 2. Serial position curves with guesses (upper panel) and without guesses (lower panel) in Experiment 1. U, ungrouped; G, grouped.

sibility, a further analysis was restricted to items transposed from the group of three to the group of four; comparing whether Item 3 was recalled in Position 6 or Position 7 of the grouped 3-4 condition and whether Item 7 was recalled in Position 3 or Position 4 of the grouped 4-3 condition. A direct test of the proportion of errors that were relative versus absolute interpositions, including guesses and collapsing across the two styles of grouping, revealed that the incidence of relative interpositions ( $M=.19, S D=.12$ ) was still significantly greater than that of absolute interpositions $[M=.12, S D=.08$;
$Z(23)=3.29, p<.001]$. Thus, the present results cannot be attributed to a bias in the baseline probability of absolute interpositions.

Between-group repetitions. The previous analyses demonstrated that a transposition at the end of one group was more likely to come from the end of the other group than from the third position of the other group, at least in the grouped 3-4 condition. Closer inspection of the data revealed that many of these errors occurred at the end of the second group and were repetitions of an item recalled at the end of the first group. In the grouped 3-4 condi- 
Table 1

Proportion of Errors on Critical Positions

That Were Interpositions in Experiment 1

\begin{tabular}{|c|c|c|c|c|c|c|c|c|}
\hline \multirow[b]{3}{*}{ Condition } & \multicolumn{4}{|c|}{ Grouped } & \multicolumn{4}{|c|}{ Ungrouped } \\
\hline & \multicolumn{2}{|c|}{ Third } & \multicolumn{2}{|c|}{ Final } & \multicolumn{2}{|c|}{ Third } & \multicolumn{2}{|c|}{ Final } \\
\hline & $M$ & $S D$ & $M$ & $S D$ & $M$ & $S D$ & $M$ & $S D$ \\
\hline \multicolumn{9}{|l|}{ Grouped 3-4 } \\
\hline Guesses includ & .12 & .09 & .18 & .13 & .15 & .07 & .17 & .09 \\
\hline Guesses excluded & .12 & .09 & .20 & 18 & .18 & .13 & .18 & .13 \\
\hline \multicolumn{9}{|l|}{ Grouped 4-3 } \\
\hline Guesses includs & .13 & .09 & .15 & .10 & .17 & .09 & .18 & .09 \\
\hline Guesses excluded & .13 & .12 & .16 & .15 & .18 & .13 & .18 & .12 \\
\hline
\end{tabular}

Note-Calculated from weighted log-odds. $N=22$.

tion, for example, Item 3 was sometimes recalled on both Position 3 and Position 7. More important, even if a different item was recalled on Position 3, that item was likely to be recalled again on Position 7 (e.g., Item 2 might be recalled on both Position 3 and Position 7), an occurrence that would have been missed by the above analyses. These errors suggest that many interpositions might be perseverations resulting from proactive interference from recall of the first group on recall of the second, much like the proactive interference found between recall of successive lists (Henson, 1996). Consequently, responses in the second group were examined in more detail.

Given a response on a critical position in the second group that was a repetition of an item recalled somewhere in the first group (a between-group repetition), the interest was whether that item came from the same critical position of the first group. For this analysis, the two grouped conditions were collapsed together, and, as before, the ungrouped lists were treated as if they were grouped in the corresponding manner. Given the small numbers involved, guesses were included, and the analysis was restricted to the 19 participants that made at least one between-group repetition per critical position.

The incidence of between-group repetitions and the proportion that were absolute or relative interpositions are shown in Table 2. Pairwise comparisons of weighted log-odds showed that a significantly greater proportion of between-group repetitions were interpositions between final positions than between third positions of groups in grouped lists $[Z(19)=2.68, p<.01]$, but not in ungrouped lists $[Z(19)=0.08, p=.94]$. The difference in proportions in grouped lists was not due to differences in the overall incidence of between-group repetitions, which did not approach significance $[Z(19)<0.45, p\rangle$ $.65]$. The incidence of repetitions between the ends of groups was also compared with that expected by chance. The chance probability that a between-group repetition will be scored as a relative interposition is difficult to determine exactly, because there are four items in the first group of the grouped 4-3 condition and three in the first group of the grouped 3-4 condition, meaning that the chance probability lies somewhere between .25 and .33 . Taking an average value of .29 (a value close to that in the ungrouped lists), repetitions between the final posi- tions of groups were more frequent than was expected by chance in the grouped lists $[Z(19)=2.82, p<.005]$, but not in the ungrouped lists $[Z(19)=0.65, p=.52]$.

Finally, interpositions were still found between the start of groups. The proportion of between-group repetitions that respected relative position $(M=.48, S D=$ .27) was significantly greater than (1) the proportion that respected absolute position $(M=.31, S D=.22),(2)$ the baseline figure in ungrouped lists $(M=.37, S D=.28)$, and (3) the figure of .29 expected by chance $[Z(16)>$ $2.03, p<.05\}$, in all cases. In other words, repetitions between groups respected both terminal positions, the start and the end, of groups.

\section{Discussion}

The present experiment showed that interpositions between groups tend to respect their relative position within groups, rather than their absolute position within groups. Although differences were small, the proportion of errors that were interpositions was greater between the ends of groups than between the third positions of groups, particularly in the grouped 3-4 condition. Similarly, the proportion of repetitions in the second group that were interpositions from the same relative position in the first group was significantly greater than the proportion from the same absolute position in the first group and was well above chance levels. These data suggest that position within a group is coded with respect to both the start and the end of that group, favoring models that code position in relative (see, e.g., Henson, 1998b), rather than in absolute (see, e.g., Anderson \& Matessa, 1997; Brown et al., in press; Burgess \& Hitch, 1992, 1996; Neath \& Crowder, 1996) terms.

The proportion of errors that were interpositions was little changed by the removal of guesses, which showed no interaction with grouping or critical position. This implies that interpositions are not simply the result of guessing strategies. The difference in the incidence of relative and absolute interpositions in grouped lists seemed to reflect a decrease in absolute interpositions, relative to ungrouped lists, rather than an increase in relative interpositions. The decrease in transpositions between the same absolute position within groups resembles the general decrease in transpositions between groups that is found when lists are grouped (Henson, 1996). The lack of a significant increase in relative interpositions was sur-

Table 2

Frequency of Between-Group Repetitions, Including Guesses, and Proportion That Were Interpositions on Critical Positions of Second Group in Experiment 1

\begin{tabular}{|c|c|c|c|c|c|c|c|c|}
\hline \multirow[b]{3}{*}{ Condition } & \multicolumn{4}{|c|}{ Grouped } & \multicolumn{4}{|c|}{ Ungrouped } \\
\hline & \multicolumn{2}{|c|}{ Third } & \multicolumn{2}{|c|}{ Final } & \multicolumn{2}{|c|}{ Third } & \multicolumn{2}{|c|}{ Final } \\
\hline & $M$ & $S D$ & $M$ & $S D$ & $M$ & $S D$ & $M$ & $S D$ \\
\hline $\begin{array}{l}\text { Between-group } \\
\text { repetitions }\end{array}$ & .16 & .07 & .17 & .08 & .20 & .08 & .19 & .08 \\
\hline Interpositions & .24 & .19 & .40 & .24 & .31 & .19 & .31 & .18 \\
\hline
\end{tabular}

Note-Calculated from weighted log-odds. $N=19$. 
prising, given that grouping also increases the incidence of interpositions (Henson, 1996; Wickelgren, 1967). When analysis was confined to between-group repetitions however, grouping was found to increase the proportion of repetitions that were relative interpositions, as well as decreasing the proportion that were absolute interpositions.

The observation that many interpositions were repetitions of an item at the end of both groups suggests that interpositions may be the result of proactive interference from recall of the first group on recall of the second. Indeed, approximately one half of the interpositions measured on critical positions were repetitions, which is probably sufficient to explain the differences between grouped and ungrouped conditions in Table 2 . In other words, interpositions across groups may result from the same output effects that cause protrusions across trials (Henson, 1996). One difference between repetitions across trials and repetitions across groups, however, is that repetitions across groups may contend with the additional effect of response suppression.

Response suppression is the suppression of previous responses that is assumed by nearly every model of serial recall, in order to explain why erroneous repetitions are so rare (e.g., Burgess \& Hitch, 1992; Henson, Norris, Page, \& Baddeley, 1996; Lewandowsky \& Li, 1994; Vousden \& Brown, 1997). It also has independent support from the fact that people occasionally fail to recall the second occurrence of a repeated item (the Ranschburg effect; Henson, 1998a; Jahnke, 1969). Response suppression is not complete, however, and is often assumed to wear off over time (Henson, 1998b). This means that repetitions, although rare, may still arise if an item is cued strongly enough to overcome its previous suppression. It is the refractory nature of suppression that can explain why relative interpositions were more frequent in the grouped 3-4 condition than in the grouped 4-3 condition: When an item is recalled at the end of the first group, there is more time for suppression to wear off before the item is cued again at the end of the second group in the grouped 3-4 condition, with three intervening responses, than in the grouped 4-3 condition, with only two intervening responses.

\section{EXPERIMENT 2}

The previous experiment demonstrated that transpositions between groups of different size tend to maintain relative rather than absolute position. The present experiment tests whether the same is true of intrusions between trials of different length. Specifically, in one condition, the participants saw five, six, or seven words on a given trial (the variable condition). In the other condition, the participants always saw six words on each trial (the fixed condition). The critical positions were the fifth position in reports and the final position in reports. In the variable condition, absolute protrusions were items on the fifth position of a report that also occurred on the fifth position of the previous report (given that protrusions are more often from the previous report than from the previous list; Henson, 1996); relative protrusions were items on the final position of a report that also occurred on the final position of the previous report. In the fixed condition, measurements of absolute and relative protrusions were, of course, equivalent. Nonetheless, the incidence of protrusions on the fifth position and final position were calculated for comparison.

The variable condition was interesting for a further reason. Precautions were taken to ensure that the participants in this condition did not know in advance the length of the list on each trial. This raises questions about the mechanism of coding relative position in short-term memory. If the end of a list is not known until it occurs, it is difficult to imagine how the positions of items could be coded relative to that end. For example, any interpretation in terms of an end marker's strength representing the degree of expectation for the end of a sequence (Henson, 1998b) would be particularly difficult to maintain.

Finally, in Experiment 2, the same method of distinguishing confident responses from guesses as that in Experiment 1 was used. There is evidence to suspect that protrusions might disappear when guesses are removed from reports. For example, Dillon and Thomas (1975) showed that participants were less confident of intrusions than of other types of error. Bjork and Healy (1974) found a similar result, provided intrusions were not phonological confusions. Dillon and Thomas, like Conrad (1960), used their results to argue that proactive interference makes correct items inaccessible and that it is only when participants subsequently resort to guessing that intrusions arise. They argued against the notion, in many models (e.g., Brown et al., in press; Burgess \& Hitch, 1992; Henson, 1998b), that proactive interference acts through response competition. If these authors are correct, the removal of guesses in the present experiment should affect protrusions to a greater extent than other types of error.

\section{Method}

Participants. Thirty students from Cambridge University were tested, 20 male and 10 female, with a mean age of 20 years.

Materials. Two blocks of $\mathbf{4 6}$ lists were constructed, each list containing five, six, or seven single-syllable words. Each word had five letters, a Kučera-Francis (1967) frequency of between four and five, and both a unique vowel sound and a unique first letter. Half of the lists had words drawn from the set yacht, goose, verve, psalm, wedge, haunt, and clump. The other half had words drawn from the set kneel, midge, latch, shine, bathe, flown, and trout. These sets were alternated across trials, so that no word appeared in two successive trials. This was to ensure that an intrusion from the preceding trial could be clearly identified. The order of words within lists was randomized, except for the constraint that, over all trials, each word appeared approximately equally often at each position.

Procedure. The blocks were assigned to two conditions in a counterbalanced fashion. The first list of six items in each block was not analyzed. In the fixed condition, the remaining 45 lists also contained six words. In the variable condition, there were 15 lists of five words, 15 of six words, and 15 of seven words. The order of 
lists in the variable condition was such that no two consecutive trials contained lists of the same length.

Each word was presented in the center of a VDU, replacing the previous word, at a rate of just over one a second ( $600 \mathrm{msec}$ on, $200 \mathrm{msec}$ off). The participants were instructed to read the words in silence. A short time after the last word had disappeared, a cue appeared, to signal immediate serial recall. In order to equate the total presentation time for each list length, this cue appeared $200 \mathrm{msec}$ after the offset of the last word in seven-item lists, $1,000 \mathrm{msec}$ after in six-item lists, and $1,800 \mathrm{msec}$ after in five-item lists. ${ }^{3}$

The instructions for recall were the same as those in Experiment 1 , with the confidence of each response being indicated via two rows of boxes on the response sheet. The number of boxes in each row always equaled the number of words that were presented in that trial, and all 14 words in the experimental vocabulary were permanently on display. Unlike Experiment 1, however, there was a separate response sheet for each trial. Initially, all the response sheets were facedown in a pile on the left of the participant. When the participants saw the cue for recall, they turned over the top response sheet from the pile, wrote their responses, and then put the response sheet facedown in a pile on their right. In this way, the participants did not know in advance the length of the list on a given trial in the variable condition and could never see the responses they had given in the previous trial.

The participants received eight practice trials, two of five words, two of six words, two of seven words, and two of six words, in that order. The order of the blocks and of the fixed and variable conditions that followed were counterbalanced across participants. The experiment lasted $45 \mathrm{~min}$.

\section{Results}

In brief, relative protrusions were more frequent than absolute protrusions in both the variable and the fixed conditions, regardless of whether guesses were included or excluded. In fact, the proportion of errors that were relative protrusions increased when guesses were removed. The results were generally a close analogue to those of Experiment 1.

Overall performance. A greater proportion of sixitem lists were correct in the fixed condition $(M=.58$, $S D=.28)$ than in the variable condition $(M=.45, S D=$ .31 ), a difference that was significant under a test of weighted $\log$-odds $[Z(30)=4.65, p<.0001]$. As was expected, the corresponding proportion was higher for five-item lists $(M=.80, S D=.20)$ and lower for sevenitem lists $(M=.20, S D=.20)$ in the variable condition. The advantage of fixed-length lists was apparent over all positions (upper panel of Figure 3); an advantage that may be attributable to spontaneous grouping in threes, for which there was more evidence in the fixed than in the variable condition. Guesses made up approximately $20 \%$ of the responses ( $65 \%$ of the errors), on average, and removing guesses affected all positions. Interestingly, recency remained strong for all the list lengths in the variable condition, even though the last item was not known in advance (lower panel of Figure 3), in agreement with Bunt (1976).

Errors on critical positions. The proportions of errors on a critical position that were protrusions from the same critical position in the previous report are shown in Table 3 for the 17 participants who made at least one error per critical position, even with guesses excluded. A three-way repeated measures ANOVA on empirical logodds showed significant effects of position [fifth vs. final; $F(1,16)=17.57, p<.001]$ and of guesses $[F(1,16)=$ $7.00, p<.05]$, but not of condition $[F(1,16)=1.92, p=$ .19]. The interaction between position and guesses approached significance $[F(1,16)=4.22, p=.06]$, but the other interactions did not $[F(1,16)<1.76, p>.20]$. The effect of position reflected the finding that protrusions were more frequent on final than on fifth positions. The effect of excluding guesses was to increase the incidence of protrusions on final positions. This suggests that the participants were more confident of an intrusion from the same relative position in the previous report than of other types of error. 4

As with Experiment 1, one might object that the inclusion of items from the report on one trial that intruded into a shorter report on the next trial is not a fair test of the coding of absolute position, because an item coded in, say, the seventh position in a list of seven has no corresponding seventh position in a list of five in which to intrude. To counter this possibility, a further test of relative versus absolute protrusions was confined to trials in which a short report was followed by a longer report (i.e., comparing protrusions from Position 5 in a list of five to Position 5 or Position 6 in a list of six, from Position 5 in a list of five to Position 5 or Position 7 in a list of seven, and from Position 6 in a list of six to Position 6 or Position 7 in a list of seven). Including guesses and excluding the 1 participant who made no errors on the critical positions, the proportion of errors in the variable condition that were relative protrusions $(M=.23, S D=.16)$ was still significantly greater than the proportion that were absolute protrusions $[M=.13, S D=.11 ; Z(29)=$ $3.26, p<.001]$, confirming that the present results are not due to a bias in the baseline probability of absolute protrusions.

Immediate intrusions. The previous analyses showed that the proportion of errors that were protrusions was greater between final positions than between fifth positions of reports. However, there were also fewer errors on final positions than on fifth positions (Figure 3), potentially affecting the proportion that were protrusions, and many participants did not make any errors on critical positions once guesses were removed. To address these problems and compare the incidence of protrusions with chance levels, the following analyses restricted errors to immediate intrusions from the preceding report for the 22 participants who made at least one such intrusion per critical position with guesses included.

The incidence of immediate intrusions and the proportion that were protrusions are shown in Table 4. Despite the fact that no item occurred in two successive lists, approximately one fifth of the errors were items occurring in two successive reports. In both conditions, such intrusions on the final position of a report had approximately a $40 \%$ probability of also occurring on the final position of the previous report. Pairwise comparisons of weighted log-odds showed that a significantly greater pro- 

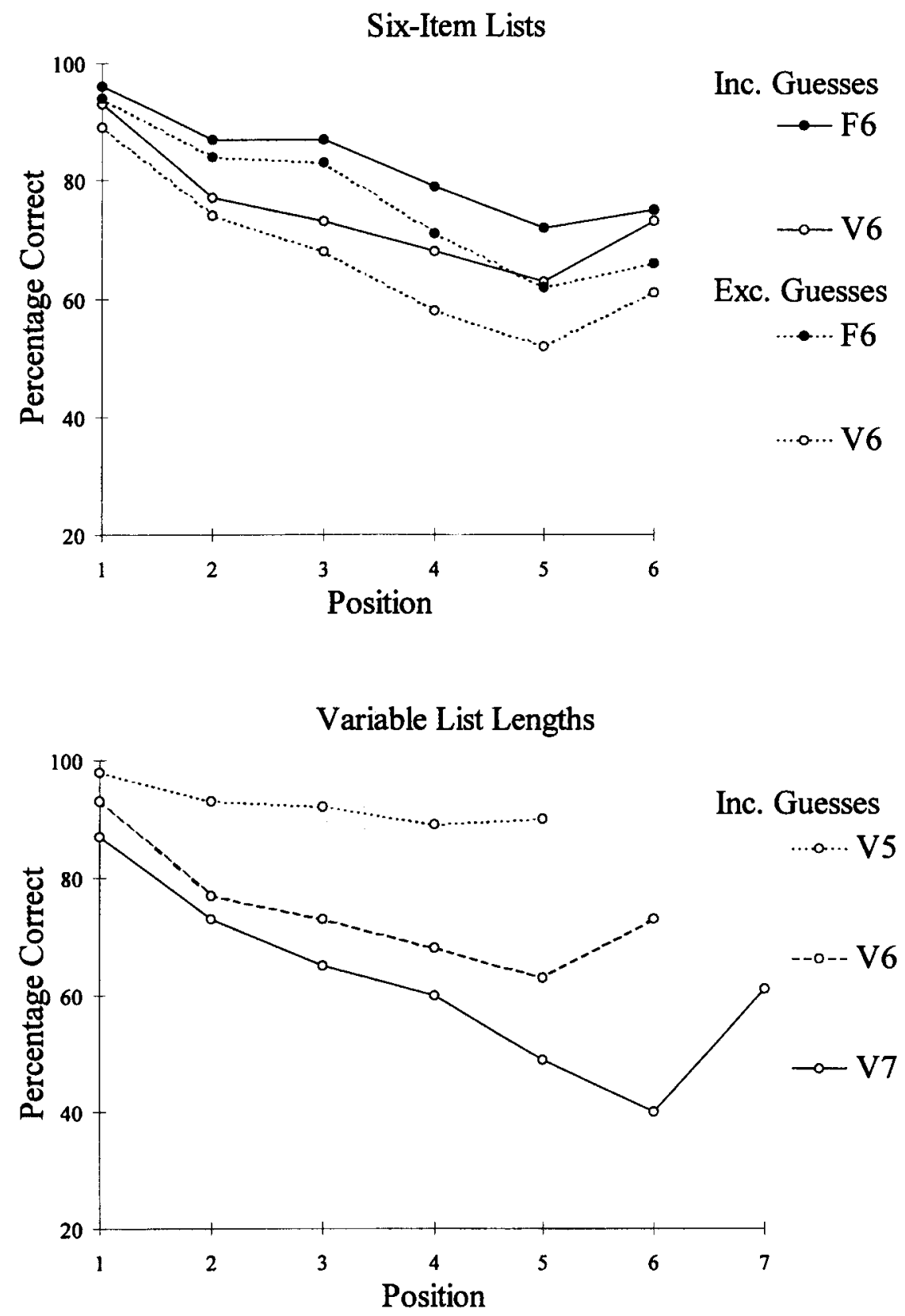

Figure 3. Serial position curves for six-item lists in the fixed and variable conditions (upper panel) and five-, six-, and seven-item lists in the variable condition (lower panel) of Experiment 2. F, fixed; $V$, variable.

portion of immediate intrusions were protrusions between final positions than between fifth positions in both conditions $[Z(22)>2.44, p<.05]$. These differences were not due to differences in the overall incidence of immediate intrusions, which showed no such significant differences $[Z(22)<1.42, p>.15]$.

The proportion of immediate intrusions that were protrusions was also compared with that expected by chance. In the fixed condition, the chance proportion was .17 (given that an intruding item could come from one of six positions in the previous report); the proportion of protrusions was significantly greater than this on both critical positions $[Z(22)>4.75, p<.0001]$. In the variable condition, the chance proportion was not so clear (given that the previous report could contain five, six, or seven items). Even taking a conservative estimate of chance of .20 (as if the previous report only contained five items), the proportion of protrusions on both the fifth position and the final position was still greater than chance $[Z(22)=$ $2.15, p<.05$ and $Z(22)=5.03, p<.0001$, respectively]. 
Table 3

Proportion of Errors on Critical Positions

That Were Protrusions in Experiment 2

\begin{tabular}{|c|c|c|c|c|c|c|c|c|}
\hline \multirow[b]{3}{*}{ Condition } & \multicolumn{4}{|c|}{ Variable } & \multicolumn{4}{|c|}{ Fixed } \\
\hline & \multicolumn{2}{|c|}{ Fifth } & \multicolumn{2}{|c|}{ Final } & \multicolumn{2}{|c|}{ Fifth } & \multicolumn{2}{|c|}{ Final } \\
\hline & $M$ & $S D$ & $\bar{M}$ & $\overline{S D}$ & $M$ & $\overline{S D}$ & $M$ & $S D$ \\
\hline$\overline{\text { Guesses included }}$ & .13 & .09 & .20 & .14 & .16 & .13 & .22 & .14 \\
\hline Guesses excluded & .12 & .13 & .26 & .25 & .16 & .17 & .32 & .24 \\
\hline
\end{tabular}

Note-Calculated from weighted log-odds. $N=17$.

Finally, protrusions on the first position of a report in the variable condition were still predominantly from the first position of the previous report. Indeed, as a proportion of immediate intrusions $(M=.57, S D=.34)$, they were significantly more frequent than the chance level of $.20[Z(23)=5.51, p<.0001]$. In other words, protrusions respected both terminal positions, the start and the end, of reports.

\section{Discussion}

The present experiment showed that protrusions between reports respect relative position, rather than absolute position. This was demonstrated in the variable condition, where the proportion of errors that were protrusions was significantly greater between the final positions of reports than between the fifth positions of reports, even when the length of lists varied from trial to trial in an unpredictable manner. Similar results have been found in two unpublished experiments analyzed by the author. These results suggest that position in a list is coded relative to both the start and the end of that list, again supporting the prediction of some models (e.g., Henson, 1998b), but not of others (e.g., Anderson \& Matessa, 1997; Brown et al., in press; Burgess \& Hitch, 1992, 1996; Neath \& Crowder, 1996).

Protrusions were also more probable between the final position of reports than between the fifth position of reports in the fixed condition. This does not invalidate the conclusions drawn from the variable condition: At least one model predicts exactly this pattern, with the higher incidence of positional errors on terminal than on preterminal positions being due to more precise coding of terminal positions (Henson, 1998b). This model also explains why the probability of absolute protrusions, although less than that of relative protrusions, was still greater than chance in the variable condition: There is still considerable similarity between the code for the fifth position in, say, a list of seven items and the code for the fifth position in a list of five items (see, also, Henson, 1996).

The present results show that protrusions are not simply the result of guessing strategies. One reason why significant numbers of protrusions remained in the absence of guesses may be that some participants were not bothering to indicate all their guesses. Nonetheless, the fact that the proportion of errors that were protrusions actually increased with the removal of guesses, at least on terminal positions, suggests that intrusions that maintain relative position were less likely to be guesses than other types of error. This supports the competition for output between items coded in similar positions that is assumed by many models of serial recall (e.g., Brown et al., in press; Burgess \& Hitch, 1992; Henson, 1998b).

Somewhat ironically, however, the confirmation of relative protrusions in a situation in which the end of the list was unpredictable raises an important question regarding the coding of relative position: How are the positions of items coded relative to the end of a sequence when that end is not known in advance? This issue is resumed in the General Discussion section.

\section{GENERAL DISCUSSION}

The present experiments demonstrated that substitutions between sequences of different lengths tend to maintain relative rather than absolute position, whether those sequences are groups within a trial (Experiment 1) or reports on different trials (Experiment 2 ). These findings are difficult to explain by models in which positional codes vary constantly over time (e.g., Brown et al., in press; Neath \& Crowder, 1990, 1996) or position (e.g., Anderson \& Matessa, 1997; Burgess \& Hitch, 1992). Although some models that code position temporally might explain end-to-end substitutions by assuming that position is defined by the time since the end of a sequence (e.g., Neath \& Crowder, 1990), the same assumption would not explain why start-to-start substitutions were still found between sequences of different length. Rather, the present findings suggest that position is coded with respect to both the start and the end of a sequence, consistent with the model of Henson (1998b). The relevant assumptions of this model are summarized below, followed by issues concerning the coding of relative position, its generality, and its relationship to temporal factors.

\section{Start and End Markers}

Following the ideas of Houghton (1990), the start-end model of Henson $(1996,1998 \mathrm{~b})$ assumes that position is coded via markers at the start and end of a sequence. The start marker is strongest at the start of a sequence but decreases in strength toward the end of the sequence; the end marker is weakest at the start but increases in strength toward the end. The relative strengths of the start and end markers therefore provide an approximate

Table 4

Frequency of Immediate Intrusions, Including Guesses, and Proportion That Were Protrusions in Experiment 2

\begin{tabular}{|c|c|c|c|c|c|c|c|c|}
\hline \multirow[b]{3}{*}{ Condition } & \multicolumn{4}{|c|}{ Variable } & \multicolumn{4}{|c|}{ Fixed } \\
\hline & \multicolumn{2}{|c|}{ Fifth } & \multicolumn{2}{|c|}{ Final } & \multicolumn{2}{|c|}{ Fifth } & \multicolumn{2}{|c|}{ Final } \\
\hline & $M$ & $S D$ & $M$ & $\overline{S D}$ & $M$ & $S D$ & $M$ & $S D$ \\
\hline $\begin{array}{l}\text { Immediate } \\
\text { intrusions }\end{array}$ & .20 & .0 & & .08 & .21 & .09 & .20 & .09 \\
\hline Protrusions & .27 & .19 & .37 & .23 & .30 & .23 & .42 & .24 \\
\hline
\end{tabular}

Note-Calculated from weighted log-odds. $N=22$. 
two-dimensional code for each position in the sequence. In other words, the start and end markers function as anchor points by which the items of a sequence can be ordered. 5

The assumption that the last items in sequences share an association with a weak start marker and a strong end marker can explain the positional errors between the end of groups and the end of reports. Likewise, an association with a strong start marker and a weak end marker can explain the positional errors between the starts of such sequences, and an association with comparable start and end marker strengths can explain positional errors between the middles of such sequences (see note 5). Simulations and further details of this model are given in Henson (1996, 1998b).

Nonetheless, the psychological interpretation of start and end markers remains unclear, particularly that of the end marker, given that items are assumed to be coded relative to the end of a sequence even when the end of that sequence has not yet occurred. One possibility is that the strength of the end marker corresponds to the degree of expectation for the end of a sequence. This interpretation is plausible for the grouped conditions of Experiment 1, where participants knew the size of both groups in advance. In this case, the strength of the end marker at the third position in a group of three, where the final item is expected, will differ from its strength at the third position in a group of four, where the final item is not yet expected. However, the expectation interpretation is less plausible when applied to the variable condition of Experiment 2 , in which the lengths of the lists were not known in advance. The finding that protrusions still maintained relative rather than absolute position makes interpretation of the end marker even more puzzling.

One possibility is that the positional codes are only generated during rehearsal, after presentation of a sequence has finished. The advantage of this possibility is that the length of the sequence is then known and an expectancy interpretation of the end marker strength can be maintained. This rehearsal hypothesis might also explain why positional errors between sequences are clearest when scored by output position rather than by input position (Henson, 1996) - in other words, when the previous sequence represents a rehearsal episode rather than a presentation episode (e.g., recall of the previous group in Experiment 1 or recall of the previous list in Experiment 2). The disadvantage of the hypothesis is that it begs the question of how the order of items is stored before rehearsal begins: If positional codes are only established during rehearsal, they cannot be used to order the very first rehearsal. Moreover, there is some evidence against this rehearsal hypothesis. For example, several studies have demonstrated that people extract positional information even under incidental learning (e.g., Hintzman, Block, \& Summers, 1973; Nairne, 1992; Toglia \& Kimble, 1976), although perhaps not as well as under intentional learning (Tzeng, Lee, \& Wetzel, 1979). Also, in a condition in which participants rehearsed lists overtly during the retention interval, Estes (1991) showed that the majority of items that did not occur in the rehearsal protocol could still occur at or near the correct position in recall. Estes therefore assumed that positional information can be generated during presentation, as well as during rehearsal, contrary to the rehearsal hypothesis. Finally, Page and Norris (1997) found further evidence against the rehearsal hypothesis by showing that the incidence of positional errors increased when presentation and recall were accompanied by concurrent articulatory suppression, which is assumed to preclude rehearsal (Baddeley, 1986).

Assuming that positional codes can be generated during presentation as well as during rehearsal, another possibility is that the end marker does not grow in strength until the very last item, when the end of a sequence is finally confirmed. In other words, the end marker might be restricted to coding the last item in a sequence. ${ }^{6} \mathrm{~A}$ related suggestion was made by Houghton (1990), whose end marker was only triggered during presentation by the termination of a sequence. However, by further assuming that the presentation of items leaves them transiently activated in memory, the triggering of the end marker allows it to take a "snap-shot" of a recency gradient of decaying activations of the last few items in the list. By growing in strength more gradually during recall, the end marker is thus able to exert an influence on items earlier in the list.

A final possibility is that, when the length of a sequence is unknown, the end marker strength might grow in a fixed manner, independent of exact sequence length. During recall, however, when the length of the sequence is known (particularly if the correct number of boxes are provided for recall), the end marker can behave as was previously assumed, growing more precise toward the end of recall, reflecting a more accurate expectation for the end of the report. Because the end marker behaves differently during presentation and recall, there will be a greater uncertainty in the cuing of preterminal items (Henson, 1996), concordant with the inferior recall of six-item lists in the variable than in the fixed conditions of Experiment 2. However, because considerable similarity will remain between the cue for the last position and the item recoded in the last position of the previous report, protrusions will still be more likely between relative positions than between absolute positions, when measured with respect to output position (for further discussion of this possibility, see Henson, 1996).

In summary, several means of coding relative position during presentation as well as during rehearsal can be hypothesized. In particular, it is possible to maintain an expectancy interpretation of the end marker by assuming a hazy notion of expectancy during presentation that is refined during recall. However, further work is necessary in order to distinguish this approach from other possible interpretations of an end marker.

\section{Sensitivity to Temporal Factors}

Another question concerns whether the coding of position in short-term memory is sensitive to temporal fac- 
tors, such as the timing of sequence presentation. In one sense, it clearly is, for the start and the end of a sequence are often defined temporally (e.g., the definition of the start and the end of groups by a pause between groups). A more difficult question is whether the coding of position within a sequence depends on temporal factors, such as presentation rate. Prima facie, any influence of such temporal factors would be easier to explain with an absolute than with a relative coding of position.

However, the demonstration of temporal influences on positional coding is not a simple matter. One cannot simply compare serial recall of sequences presented rapidly with sequences presented slowly, because participants may rehearse sequences at a rate that differs from the objective presentation rate. Baddeley and Lewis (1984) overcame this problem by preventing rehearsal with concurrent articulatory suppression. In this case, slower recall resulted in worse serial recall (see, also, Shiffrin \& Cook, 1978). However, a problem with this approach is that slower presentation rates also entail a longer interval between presentation and recall of each item, which may impair recall for reasons other than the coding of position (such as the decay of phonological information in short-term memory; Baddeley, 1986).

Better evidence for an effect of temporal factors that cannot be attributed to retention interval comes from experiments by Neath and Crowder $(1990,1996)$. These authors compared presentation schedules in which the temporal spacing between items either increased or decreased over successive positions, so that total presentation time was equated. Their data suggested that when participants recall serially (from the first through to the last item), performance is better under an increasing than under a decreasing schedule. This finding was used to support a theory of temporal distinctiveness in which items are ordered directly by their time of occurrence (i.e., an absolute coding of position).

However, evidence of this type is not incompatible with a relative coding of position. For example, if the rates of change of the strengths of start and end markers in the start-end model (Henson, 1998b) are made a function of time, the model also becomes sensitive to different presentation schedules. Indeed, it is not difficult to construct other representations of relative position that are consistent with Neath and Crowder's $(1990,1996)$ findings, yet do not assume an absolute coding of time of occurrence (see Henson \& Burgess, 1997). Thus, an important area for future research will be to specify more precisely the temporal factors that affect the coding of position and to develop theories that combine evidence from positional errors between sequences with evidence from different temporal presentation schedules within sequences.

\section{Conclusion}

Two experiments demonstrated that positional errors between sequences of different lengths respect both the start and the end of those sequences and that such errors are not simply position-sensitive guesses. These results are consistent with models that code position via start and end markers, yet raise problems for most other models of serial recall. Nonetheless, the demonstration of positional errors between the ends of sequences when the ends of those sequences are unpredictable prevents any simple interpretation of an end marker. Further research may help clarify the nature of end markers by testing (1) whether position is really coded during presentation in the absence of rehearsal and (2) whether position is truly relative, extending to more than just terminal positions. Further research is also needed to clarify the relationship between the temporal characteristics of sequences and the coding of relative position within those sequences.

\section{REFERENCES}

Allerup, P., \& Elbro, C. (1998). Comparing differences in accuracy across conditions or individuals: An argument for the use of log-odds. Quarterly Journal of Experimental Psychology, 51A, 409-424.

ANDERSON, J. R., \& MATESSA, M. (1997). A production system theory of serial memory. Psychological Review, 104, 728-748.

BADDEleY, A. D. (1986). Working memory. Oxford: Oxford University Press, Clarendon Press.

BADDELEY, A. D., \& LEWIS, V. J. (1984). When does rapid presentation enhance digit span? Bulletin of the Psychonomic Society, 22, 403-405.

Badpeley, A. D., Thomson, N., \& Buchanan, M. (1975). Word length and the structure of short-term memory. Journal of Verbal Learning \& Verbal Behavior, 14, 575-589.

BJorK, E. L., \& HEALY, A. F. (1974). Short-term order and item retention. Journal of Verbal Learning \& Verbal Behavior, 13, 80-97.

Brown, G. D. A., Preece, T., \& Hulme, C. (in press). Oscillator-based memory for serial order. Psychological Review.

Brown, G. D. A., \& Vousden, J. I. (1998). Adaptive analysis of sequential behaviour: Oscillators as rational mechanisms. In M. Oaksford \& N. Chater (Eds.), Rational models of cognition. Oxford: Oxford University Press.

Bunt, A. A. (1976). Context effects in short-term memory: Confirmatory evidence from recall of visually presented lists. Acta Psychologica, 40, 423-430.

Burgess, N., \& HITCH, G. (1992). Toward a network model of the articulatory loop. Journal of Memory \& Language, 31, 429-460.

Burgess, N., \& Hitch, G. (1996). A connectionist model of STM for serial order. In S. Gathercole (Ed.), Models of short-term memory (pp. 51-71). Hove, U.K.: Psychology Press.

ConRaD, R. (1960). Serial order intrusions in immediate memory. British Journal of Psychology, 51, 45-48.

Cox, D. R., \& SNELL, E. J. (1989). Analysis of binary data (2nd ed.). London: Chapman \& Hall.

Dillon, R. F., \& Thomas, H. (1975). The role of response confusion in proactive interference. Journal of Verbal Learning \& Verbal Behavior, 14, 603-615.

EsTES, W. K. (1991). On types of item coding and sources of recall in short-term memory. In W. E. Hockley \& S. Lewandowsky (Eds.), Relating theory and data: Essays on human memory in honor of Bennet B. Murdock (pp. 175-194). Hillsdale, NJ: Erlbaum.

FLEISS, J. L. (1981). Statistical methods for rates and proportions. New York: Wiley.

HENSON, R. N. A. (1996). Short-term memory for serial order. Unpublished doctoral dissertation, University of Cambridge.

HENSON, R. N. A. (1998a). Item repetition in short-term memory: Ranschburg repeated. Journal of Experimental Psychology: Learning, Memory, \& Cognition, 24, 1162-1181.

Henson, R. N. A. (1998b). Short-term memory for serial order: The start-end model of serial recall. Cognitive Psychology, 36, 73-137.

Henson, R. N. A., \& Burgess, N. (1997). Representations of serial 
order. In J. A. Bullinaria, D. W. Glasspool, \& G. Houghton (Eds.), 4th neural computation and psychology workshop (pp. 283-300). London: Springer-Verlag.

Henson, R. N. A., Norris, D. G., Page, M. P. A., \& Baddeley, A. D. (1996). Unchained memory: Error patterns rule out chaining models of immediate serial recall. Quarterly Journal of Experimental Psychology, 49A, 80-115.

Hintzman, D. L., BLock, R. A., \& Summers, J. L. (1973). Contextual associations and memory for serial position. Journal of Experimental Psychology, 97, 220-229.

Houghton, G. (1990). The problem of serial order: A neural network model of sequence learning and recall. In R. Dale, C. Mellish, \& $M$. Zock (Eds.), Current research in natural language generation (pp. 287-319). London: Academic Press.

JAHnKe, J. C. (1969). The Ranschburg effect. Psychological Review, 76, 592-605.

KuČERA, M., \& FranCIS, W. (1967). Computational analysis of presentday American English. Providence, RI: Brown University Press.

LEE, C. L., \& EsTES, W. K. (1977). Order and position in primary memory for letter strings. Journal of Verbal Learning \& Verbal Behavior, 16, 395-418.

LEE, C. L., \& EsTES, W. K. (1981). Item and order information in shortterm memory: Evidence for multilevel perturbation processes. Journal of Experimental Psychology: Human Learning \& Memory, 7, 149-169.

LEWANDOWSKY, S., \& LI, S. (1994). Memory for serial order revisited. Psychological Review, 101, 539-543.

LEWANDOWSKY, S., \& MURDOCK, B. B., JR. (1989). Memory for serial order. Psychological Review, 96, 25-57.

MURDOCK, B. B., JR. (1995). Developing TODAM: Three models for serial order information. Memory \& Cognition, 23, 631-645.

NAIRNE, J. S. (1991). Positional uncertainty in long-term memory. Memory \& Cognition, 19, 332-340.

NAIRNE, J. S. (1992). The loss of positional certainty in long-term memory. Psychological Science, 3, 199-202.

NeATH, I., \& Crowder, R. G. (1990). Schedules of presentation and temporal distinctiveness in human memory. Journal of Experimental Psychology: Learning, Memory, \& Cognition, 16, 316-327.

NEATH, I., \& CROWDER, R. G. (1996). Distinctiveness and very shortterm serial position effects. Memory, 4, 225-242.

PAGE, M. P. A., \& NorRIS, D. G. (1997). [A series of experiments on memory for serial order]. Unpublished raw data.

PAGE, M. P. A., \& Norris, D. G. (1998). The primacy model: A new model of immediate serial recall. Psychological Review, 105, 761-781.

RYAN, J. (1969). Grouping and short-term memory: Different means and patterns of grouping. Quarterly Journal of Experimental Psychology, 21, 137-147.

ShIFFrin, R., \& CooK, J. (1978). Short-term forgetting of item and order information. Journal of Verbal Learning \& Verbal Behavior, 17, 189-218.

TogliA, M. P., \& KimBle, G. A. (1976). Recall and use of serial position information. Journal of Experimental Psychology: Human Learning \& Memory, 2, 431-445.

Tzeng, O. J. L., Lee, A. T., \& Wetzel, C. D. (1979). Temporal coding in verbal information processing. Journal of Experimental Psychology: Human Learning \& Memory, 5, 52-64.

VousDEN, J. I., \& Brown, G. D. A. (1997). To repeat or not to repeat: The time course of response suppression in sequential behavior. In J. A. Bullinaria, D. W. Glasspool, \& G. Houghton (Eds.), 4th neural computation and psychology workshop (pp. 301-315). London: Springer-Verlag.

WICKELGREN, W. A. (1967). Rehearsal grouping and hierarchical organization of serial position cues in short-term memory. Quarterly Journal of Experimental Psychology, 19, 97-102.

YNTEMA, D. B., \& TRASK, F. P. (1963). Recall as a search process. Journal of Verbal Learning \& Verbal Behavior, 2, 65-74.

\section{NOTES}

1. Although the positional codes assumed by the perturbation model (Lee \& Estes, 1977) are not explicitly stated as absolute or relative, the mathematical formalization of the model (Lee \& Estes, 1981) would appear to assume that position is coded in absolute terms.

2. I thank an anonymous reviewer for raising this possibility.

3. A second reviewer observed that this manipulation of cue onset would also allow more rehearsals of shorter lists than of longer lists before recall. Although possible, it is not immediately apparent how this would affect the conclusion of the experiment (i.e., the comparative incidence of absolute and relative protrusions). More important, the same conclusion has since been reached after analysis of two independent experiments in which there was no such manipulation of cue onset.

4. Further analysis (Henson, 1996) showed that removing guesses did reduce the overall incidence of intrusions. That is, intrusions that were not positional were likely to be guesses, in agreement with Bjork and Healy (1974) and Dillon and Thomas (1975). One reason for this is that there may be a greater baseline chance that a random guess is an intrusion rather than a transposition, particularly if participants tend not to guess an item they have already recalled (owing to response suppression; Henson, 1996). In other words, it seems that two types of intrusion can be distinguished: those that result from competition between items at similar positions in trials (protrusions) and those that result from random guesses from the experimental vocabulary.

5. Note that the idea of start and end markers is not new: Such markers are assumed in both the model of Shiffrin and Cook (1978) and TODAM (e.g., Lewandowsky \& Murdock, 1989; Murdock, 1995). It is the use of these markers to code position that is new. The models considered by Shiffrin and Cook and Murdock are not positional models. They represent serial order in relational terms, via associations between items (either directly in the latter model or indirectly, via nodes, in the former model). The start and end markers in these models are used simply as entry points with which to retrieve the first and last items, after which other items are retrieved via the associations between them. Thus, although the models of Shiffrin and Cook and Murdock might be extended to the present findings of errors between items at the end of sequences (via associations to a common end marker), they cannot explain positional errors between items in the middle of sequences. For example, the proportion of immediate intrusions that were protrusions on Positions $2-5$ in the fixed condition of Experiment 2 was $29 \%, 27 \%, 35 \%$, and $26 \%$, respectively. Each of these values is greater than the chance level of $17 \%$ (one sixth). The only way this could be explained by the models of Shiffrin and Cook or Murdock is by intrusion of whole subsequences of items from the previous report (e.g., an end marker accidentally providing access to the last three items in the previous report). However, this is almost never the case (Henson, 1996). In the above example from Experiment 2, only $10 \%$ of the protrusions were followed by a further protrusion-a small proportion, as compared with the $41 \%$ of protrusions followed by a correct response. In other words, protrusions arise on middle positions without prior recall of preceding or succeeding items (see Lee \& Estes, 1981, and Nairne, 1991, for further discussion of the independence of positional errors).

6. The problem with parametizing the end marker of the start-end model (Henson, 1998b) to behave in such a discrete fashion is that it no longer allows the model to give such good fits to data (e.g., a gradual growth of end marker strength is better able to produce positional errors between the middle of sequences; Henson, 1996).

\section{APPENDIX \\ Logit or "Log-Odds" Transform}

If participant $i$ of $N$ makes $r_{i}$ errors in a sample of $n_{i}$ responses, giving a basic proportional score of $p_{i}=r_{i} / n_{i}$, then the log-odds score, $l_{i}$, is defined as

$$
l_{i}=\ln \left[p_{i} /\left(1-p_{i}\right)\right]=\ln \left[r_{i} /\left(n_{i}-r_{i}\right)\right] \text {. }
$$

This logarithmic transform "stretches" proportional scores close to zero and close to one, making some allowance for floor and ceiling effects. However, the transform is not defined at these extremina exactly - that is, when $r_{i}=0$ or $r_{i}=n_{i}$. 


\section{APPENDIX (Continued)}

\section{Empirical Log-Odds}

To handle situations when $r_{i}=0$ or $r_{i}=n_{i}$ (and $n_{i}>0$ ), an empirical log-odds score can be defined (Cox \& Snell, 1989):

$$
l_{i}=\ln \left[\left(r_{i}+0.5\right) /\left(n_{i}-r_{i}+0.5\right)\right] \text {. }
$$

This caters to proportions of zero or one. However, it makes no allowance for the fact that one proportional measure may be based on a large number of observations (when $n_{i}$ is large) and, hence, is likely to be more accurate than one based on a small number of observations (when $n_{i}$ is small).

\section{Weighted Log-Odds}

The empirical log-odds score defined above has an associated variance:

$$
u_{i}=\left(n_{i}+1\right)\left(n_{i}+2\right) /\left[n_{i}\left(r_{i}+1\right)\left(n_{i}-r_{i}+1\right)\right] .
$$

Empirical log-odds can be weighted by the inverse of their variances,

$$
w_{i}=1 / u_{i}
$$

to give a weighted mean across participants:

$$
L=\sum w_{i} l_{i} / \sum w_{i},
$$

where the summand is from participant $i=1$ to $i=N$.

Transforming back into the original coordinates, the estimated mean proportion over participants, $P$, has variance, $V$, given by

$$
P=\exp (L) /[1+\exp (L)] \quad V=U P^{2}(1-P)^{2},
$$

where $U$ is the mean variance of the empirical log-odds.

\section{Testing Related, Weighted Log-Odds}

To test a difference in means of two related log-odds, $l_{i}$ and $l_{i}^{\prime}$, let

$$
d_{i}=l_{i}-l_{i}^{\prime}
$$

A combined weight, $\omega_{i}$, can be determined from

$$
\omega_{i}=\left(1 / w_{i}+1 / w_{i}^{\prime}\right)^{-1},
$$

where $w_{i}$ and $w_{i}{ }^{\prime}$ are the inverse of the variances of the two logodds. The weighted mean difference score, $D$, is then

$$
D=\Sigma \omega_{i} d_{i} / \Sigma \omega_{i},
$$

and the standard error of the difference score is $\left(1 / \sum \omega_{i}\right)^{1 / 2}$, which, using the critical ratio test under the null hypothesis that the underlying proportions share a common value (Fleiss, 1981), enables testing of the standardized score:

$$
Z(N)=\Sigma \omega_{i} d_{i} /\left(\sum \omega_{i}\right)^{1 / 2}
$$

(Manuscript received June 2, 1997; revision accepted for publication July 30, 1998.) 